\title{
Hyperpolarization by DNP and Molecular Dynamics: Eliminating the Radical Contribution in NMR Relaxation Studies
}

Bulat Gizatullin, Carlos Mattea, Siegfried Stapf*

FG Technische Physik II/Polymerphysik, Technische Universität Ilmenau, D-98684 Ilmenau, Germany

\author{
Supplementary information
}




\section{Materials and methods}

The polystyrene-block-polybutadiene-block-polystyrene block copolymer (Sigma-Aldrich) with a molecular weight of $140 \mathrm{kDa}$ and $30 \%$ of styrene content was diluted in deuterated cyclohexane- $\mathrm{d}_{12}$ (99,9\%, Sigma Aldrich) to obtain an SBS concentration of 10 vol.\% and was then stirred on a vortex mixer for 2 hours at $+40^{\circ} \mathrm{C}$. The pure 10 vol. $\%$ SBS solution was filled into $10 \mathrm{~mm}$ outer diameter borosilicate tubes (Deutero $\mathrm{GmbH}$, Kastellaun, Germany) for carrying out thermal polarization reference measurements. In the preparation of the samples for DNP measurements, cyclohexane-d 12 was first mixed with radicals at defined concentrations, and subsequently employed to obtain 10 and $20 \mathrm{mM}$ of 2,2,6,6-Tetramethylpiperidine 1-oxyl (TEMPO) (98\%, Sigma-Aldrich), 2.5 and $11 \mathrm{mM}$ of $\alpha, \gamma$-bisdiphenylene- $\beta$-phenylallyl (BDPA) (SigmaAldrich), respectively, in 10 vol. \% block copolymer solution. The block copolymer solutions were then filled into $2.5 \mathrm{~mm}$ inner diameter ( 3 mm outer diameter) borosilicate glass tubes (Wilmad-LabGlass, Vineland, New Jersey, U.S.A.), and flame-sealed immediately. All samples were stored at $+5^{\circ} \mathrm{C}$ and were measured within one week of preparation, while radical concentration stability was controlled by EPR spectra measurements using a benchtop X-band EPR spectrometer Magnettech 5000 (Magnettech, Freiberg Instruments, Freiberg, Germany).

The homebuilt X-band resonator is based on a dielectric cavity resonator operating in the $\mathrm{TM}_{110}$ mode at $\sim 9.6 \mathrm{GHz}$, which is suitable for both transverse and axial magnet geometries with a bore access of at least $20 \mathrm{~mm}$. The probehead includes a planar radio frequency coil for NMR detection and is compatible with standard $3 \mathrm{~mm}$ NMR tubes. Quality factors $Q=f_{0} / \Delta f$ were obtained from reflection coefficient measurements of the critically coupled resonator, where $f_{0}$ is the resonance frequency and $\Delta f$ is the width of the resonator dip at $-3 \mathrm{~dB}$. The results are given in the Table S1. The conversion factor was estimated as $c=16 \mu \mathrm{T} / \mathrm{W}^{-0.5}$, which is used for simulation of DNP spectra and power dependencies of enhancement factor.

The advanced sequence ( see Figure S1) which combines magnetic field cycling, microwave irradiation during the polarization interval and a CPMG pulse sequence during signal acquisition was used to obtain $T_{1}-T_{2}$ map, DNP spectra and NMRD data. The hyperpolarization conditions are achieved by adjustment of polarization field (I) to follow either SE or OE at the constant frequency of the microwave field. The corresponding polarization field in the performed experiments was adjusted around $340 \mathrm{mT}(14.5 \mathrm{MHz})$, which corresponds to the value of the microwave frequency of $9.57 \mathrm{GHz}$ (X-band). The microwave irradiation is switched off with simultaneous switching of the magnetic field strength to the value of the relaxation field after reaching the equilibrium value of polarization, which consists of $\sim 5 T_{1 b}$, where $T_{1 b}$ is a build-up time ( $T_{1 b} \approx T_{1}$ at low power of microwave irradiation). No effect of microwave irradiation on measured relaxation time was expected until the case of matching of polarization field and relaxation field strength in the range corresponding to the broadness of the EPR spectra of the radical, which equals approximately $500 \mathrm{kHz}$ and $150 \mathrm{kHz}$ for TEMPO and BDPA radicals (see Figure S2) in the studied samples, respectively. The protocol of the experiment assumes excluding the point on the NMRD with corresponding matched conditions. Acquisition consisted of detection of a train of echo peak inten sities by a CPMG pulse sequence with $100 \mu$ s echo time and a $\pi$-pulse duration of $8 \mu$ s. Thus two-dimensional data sets of $3072 \times 32$ points were acquired from $T_{1}-T_{2}$ correlation measurements. The two-dimensional ILT analysis was performed using a set of $32 \times 32$ logarithmically spaced input values of relaxation times in the range of 1-10000 $\mathrm{ms}$ for both $T_{1}$ and $T_{2}$ values. The logarithmic mean values $\left\langle T_{2}\right\rangle_{i}$ obtained from $T_{1}-T_{2}$ measurements for the corresponding components were used for the $T_{2}$-resolved DNP and NMRD measurements, where the decays obtained by CPMG sequences were fitted by a sum of two exponential functions, keeping $\left\langle T_{2}\right\rangle_{i}$ relaxation times fixed, and the relative amplitude of the corresponding components as a free variable. The obtained frequency dependencies of the NMR signal amplitude of different components were recalculated by $E=P_{D N P} / P_{T P}$ to DNP spectra, where $P_{\mathrm{DNP}}$ and $P_{\mathrm{TP}}$ are NMR signals at DNP condition and thermal polarization, correspondingly. In case of $T_{2}$-resolved $T_{1}$ measurements, the measured spin-lattice relaxation curves (decays or recoveries) were fitted by an exponential function to obtain values of the $R_{1}$ relaxation rate of the corresponding component.

Pre-tuning of airflow temperature and dummy scans were used to achieve a constant temperature (293 K) of the sample during all experiments. In order to control sample temperature, comparison of the measured relaxation times under microwave irradiation and previously obtained temperature dependence of corresponding relaxation times without microwave irradiation during the polarization time interval. Preliminary tests of different recycle delay times at particular microwave power and experimental parameters were performed to eliminate microwave heating effects of the resonator itself during the polarization time. 


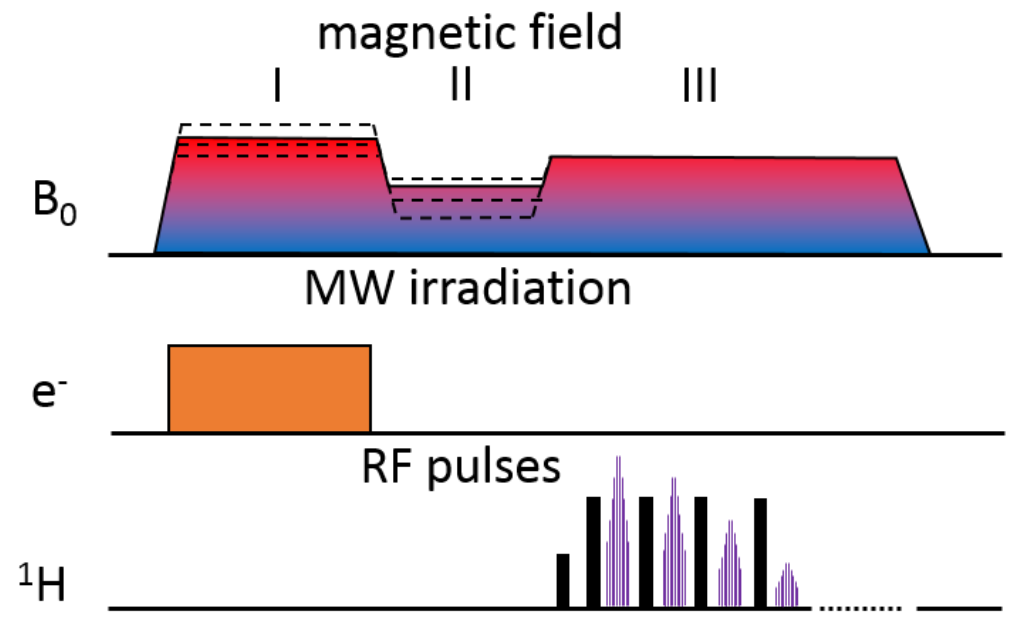

Figure S1. Pulse sequence for the measurement of $T_{1}$ relaxation dispersion profiles using hyperpolarized nuclear spin and $T_{2}$ resolving by CPMG sequences (I- polarization interval, II- relaxation interval, III- acquisition interval). The hyperpolarization/thermal polarization conditions are achieved by adjustment of polarization field (I) to follow different DNP effects, while microwave frequency remains constant. In the third interval, the CPMG RF pulse train is used to acquire NMR signal and information about transverse magnetization decay.

\section{Theory and simulation}

EPR. The EPR spectra of BDPA were fitted by a model of nine groups of electron spins, equivalent to the nine main lines of BDPA, assuming nine Voigtian line shape functions with equal Gaussian $\Gamma^{\mathrm{G}}$ and Lorentzian $\Gamma^{\mathrm{L}}$ line-broadening parameters :

$$
\begin{aligned}
& \varphi_{k}(\Delta)=\int_{-\infty}^{+\infty} \phi^{G}(x) \phi_{k}^{L}(\Delta-x) d x \\
& \phi_{k}^{L}(\Delta-x)=\frac{2}{\pi \sqrt{3}} \frac{1}{\Gamma^{L}}\left[1+\frac{4}{3}\left(\frac{\Delta-x+(k-4) A}{\Gamma^{L}}\right)^{2}\right]^{-1} \\
& \phi^{G}(x)=\sqrt{\frac{2}{\pi}} \frac{1}{\Gamma^{G}} \exp \left[-2\left(\frac{x}{\Gamma^{G}}\right)^{2}\right]
\end{aligned}
$$

where $\Gamma^{\mathrm{G}}$ and $\Gamma^{\mathrm{L}}$, respectively, are the Gaussian and Lorentzian line broadening parameters, and $A$ is the average hyperfine coupling constant. The function $\varphi_{\mathrm{k}}(\Delta)$ is normalized according to $\int_{-\infty}^{\infty} \varphi_{k}(\Delta) d \Delta=1$.

In case of TEMPO, EPR spectra were fitted with a two components model including simulation of three hyperfine lines of TEMPO by three Voigtian lines with corresponding line broadening parameters $\Gamma^{\mathrm{G}}$ and $\Gamma^{\mathrm{L}}$, which are presented in Table 1 .

DNP.

The Overhauser effect occurs when the electron spin transitions are saturated by microwave irradiation and there is hyperfine interaction between nuclear and electron spins. At the same time, there must be a process which modulates this hyperfine interaction, e.g. by molecular motions, with a rate that is sufficiently high to provide spectral components of the internal magnetic fields at the electron spin Larmor frequency. Therefore, OE is expected in comparatively mobile system such as diluted polymer solutions. The SE appears to be present not only in solids but already in fluids of rather low viscosity, such as concentrated polymer solutions or melts. The SE DNP mechanism has a maximum effect (enhancement) when the microwave and ESR frequencies differ by approximately the NMR frequency, i.e. $f_{M W}=f_{\mathrm{e}} \pm f_{\mathrm{n}}$. These conditions lead to excitation of "forbidden" zero- and double quantum electron-nuclear spin transitions which cause maximum NMR signal enhancements with inverted or non-inverted NMR signal phase at corresponded frequency. When both the homogeneous linewidth and the inhomogeneous spectral width of the EPR spectrum are small compared to the nuclear spin Larmor frequency, the well-resolved SE can be observed ${ }^{1}$. The frequency dependence of DNP enhancement, or so called DNP spectra, can be measured by sweeping the polarization field at constant microwave frequency, i.e. by changing $f_{\mathrm{e}}$, or by varying $f_{\mathrm{MW}}$ at constant polarization field strength.

For the simulation of DNP spectra a model was applied that included both OE and SE contributions. The total enhancement $E$ for the coupled electron-nuclei system can be expressed by: 


$$
E=p_{O E} E_{O E}+p_{S E} E_{S E}=\frac{p_{O E} P^{O E}+p_{S E}\left(p P_{n}^{S E}+[1-p] P_{n 0}\right)}{P_{n 0}}
$$

where $p_{\mathrm{OE}}+p_{\mathrm{SE}}=1$. $p_{\mathrm{OE}}$ and $p_{\mathrm{SE}}$ are the fractions of nuclear spins in the system which can be hyperpolarized by OE and SE, respectively. It is assumed, that inside the $p_{\mathrm{SE}}$ fraction, the hyperpolarization by SE is not complete, and the parameter $p$ accounts for the part of nuclear spins that are effectively enhanced by SE, while (1-p) is the remaining fraction that is only thermally polarized. Generally, in the low temperature approximation in solids, the fraction $p$ is close to unity due to the transfer of hyperpolarized state of nuclei by the spin diffusion process, while at high temperature the intensive molecular mobility prevents significant hyperpolarization of those nuclei farthest from radicals which necessitates considering residual parts of thermally polarized nuclear spins.

The solution for the OE is expressed ${ }^{2}$ in terms of coupling $\xi$, leakage $f$ and saturation factors $s$ :

$$
P_{n}^{O E}=\xi f s P_{e 0}+P_{n 0}
$$

where

$$
\begin{aligned}
& \xi=\frac{w_{2}^{O E}-w_{0}^{O E}}{w_{0}^{O E}+2 w_{1}^{O E}+w_{2}^{O E}} \\
& f=\frac{w_{0}^{O E}+w_{1}^{O E}+w_{2}^{O E}}{R_{1}^{O E}} \\
& S=\frac{v_{1}}{W+v_{1}}
\end{aligned}
$$

and where the actual observed relaxation rate of the nuclear part, which is enhanced by $O E, R_{1}^{\mathrm{OE}}$ is given by

$$
R_{1}^{O E}=w_{0}^{O E}+w_{1}^{O E}+w_{2}^{O E}+R_{1,0}^{O E}
$$

with $w_{0}^{\mathrm{OE}}, w_{1}^{\mathrm{OE}}$ and $w_{2}^{\mathrm{OE}}$ being the relaxation rates for zero-, single- and double-quantum transitions due to coupling with an electron spin, and $R_{1,0}^{\mathrm{OE}}$ is the nuclear spin-lattice relaxation rate measured in the absence of the radicals for the part of the system enhanced via $\mathrm{OE}$.

For the SE, the corresponding expression for nuclear polarization can be written as

$$
\begin{aligned}
& P_{n}^{S E}=\frac{\left(v_{0}-v_{2}\right) P_{e}+P_{n 0} R_{1,0}^{S E}}{R_{1,0}^{S E}+v_{0}+v_{2}} \\
& P_{e}=\frac{W}{W+v_{1}} P_{e 0}
\end{aligned}
$$

where $W=\left(2 T_{1 \mathrm{e}}\right)^{-1}$ is the transition rate related to the electron spin-lattice relaxation time $T_{1 \mathrm{e}}$, and $R_{1,0}^{S E}$ is the nuclear spin lattice relaxation rate of nuclei enhanced by $\mathrm{SE}$, which in principle equals to $R_{1}^{O E}$ in the case of one-component system. On the other hand, $R_{1,0}^{S E}$ and $R_{1}^{O E}$ in equation (S9) may be not equal if the heterogeneous system is characterized by several phases with different dynamics and NMR properties, where OE and SE are observed separately for different phases. $P_{\mathrm{e} 0}$ and $P_{\mathrm{n} 0}$ are the thermal equilibrium electron and nuclear spin polarizations, respectively, which are proportional to the corresponding gyromagnetic ratios, $P_{\mathrm{n} 0} \propto \gamma_{\mathrm{n}}$ and $P_{\mathrm{e} 0} \propto \gamma_{\mathrm{e}}$.

The transitions rates $v_{0}, v_{1}$ and $v_{2}$ induced by microwave irradiation are given by

$$
\begin{aligned}
& v_{0}(\Delta)=n^{-1} 4|q|^{2} \frac{\pi}{2}\left(\left|\gamma_{e}\right| B_{1}\right)^{2} \varphi\left(\Delta-f_{n}\right) \\
& v_{1}(\Delta)=\frac{\pi}{2}\left(\left|\gamma_{e}\right| B_{1}\right)^{2} \varphi(\Delta) \\
& v_{2}(\Delta)=n^{-1} 4|q|^{2} \frac{\pi}{2}\left(\left|\gamma_{e}\right| B_{1}\right)^{2} \varphi\left(\Delta+f_{n}\right)
\end{aligned}
$$

where $n=N_{\mathrm{n}} / N_{\mathrm{e}}$ is the ratio of the numbers of nuclear spins $N_{\mathrm{n}}$ and unpaired electron spins $N_{\mathrm{e}}, q$ denotes the states mixing coefficient as defined in literature ${ }^{3}, \varphi(\Delta)$ is the electron line shape, and $B_{1}=c\left(Q P_{M W}\right)^{-0.5}$ is the microwave magnetic field strength, which depends on the microwave power $P_{\mathrm{MW}}$, the microwave resonator's quality factor $Q$ and the microwave conversion factor $c$ which defines to what extent the resonator converts microwave power into $B_{1}$ (microwave magnetic field) at the location of the sample. Generally, the state mixing coefficient $q \ll 1$ is a factor calculated by first-order perturbation theory and defines the probability that the "forbidden" transition becomes allowed in the presence of non-averaging dipolar interaction, e.g. in viscous liquids, solids, liquid crystals etc.

NMRD. The FFHS (force-free-hard-sphere) model assuming only dipolar interactions modulated by translational diffusion ${ }^{4-6}$ was used for the analysis of frequency dependencies of the relaxation rate $r_{1, \text { rad }}(\omega)$ induced by added radicals. The FFHS model is part of a more general approach to describe relaxation due to translational and rotational diffusion developed by Hwang and Freed ${ }^{3,4}$ and is widely used for describing dipolar relaxation in liquids modulated by translational diffusion ${ }^{7,8}$. The FFHS mode assumes the absence of any "sticking" effects of radicals with respect to the nuclear spin-bearing molecules. The translational correlation time $\tau_{t}$ then is the relevant parameter in the FFHS model describing the mutual mobility of two spins ' host molecules and is defined as:

$$
\tau_{t}=\frac{2 d^{2}}{D_{n}+D_{e}}
$$


where $D_{\mathrm{n}}$ and $D_{\mathrm{e}}$ are self-diffusion coefficients of the molecules containing the nuclear and electron spins, respectively, and $d$ is the minimal distance of approach between electron and nuclei, which is assumed to be the same for all directions, i.e. the electron spin is at the center of a spherical molecule. If the electron spin relaxation times are longer than the translational correlation time, which is usually fulfilled for nitroxide radicals such as TEMPO, the reduced spectral density for the translational diffusion modulated by dipolar interaction is given by ${ }^{3,4}$ :

$$
j_{d}(u)=\frac{1+5 u / 8+u^{2} / 8}{1+u+u^{2} / 2+u^{3} / 6+4 u^{4} / 81+u^{5} / 81+u^{6} / 648}
$$

where

$$
u=\sqrt{2 \omega \tau_{t}}
$$

The spin-lattice relaxation rate which is caused by electron-nuclear dipolar interaction is then written as:

$$
\left(R_{1}\right)_{d i p} \propto\left[j_{d}\left(\omega_{e}-\omega_{n}, \tau_{t}\right)+3 j_{d}\left(\omega_{n}, \tau_{t}\right)+6 j_{d}\left(\omega_{e}+\omega_{n}, \tau_{t}\right)\right]
$$

where the three terms correspond to the zero, single and double quantum transition rates $w_{0}^{O E}, w_{1}^{O E}$ and $w_{2}^{O E}$ in equation $(\mathrm{S} 6)$. Using $\omega_{e}>>\omega_{n}$ it is possible to obtain an expression for the radical-induced relaxivity:

$$
r_{1, \text { rad }}=\hat{r}^{\text {rad }}\left[7 j_{d}\left(\omega_{e}, \tau_{t}\right)+3 j_{d}\left(\omega_{n}, \tau_{t}\right)\right]
$$

with the amplitude $\hat{r}^{\text {rad }}$ at $\omega_{e, n}=0$.

Further calculations using eqs. (S6), (S18) and (S19) lead to the following expression for the coupling factor:

$$
\xi=\frac{5 j_{d}\left(\omega_{e}, \tau_{t}\right)}{7 j_{d}\left(\omega_{e}, \tau_{t}\right)+3 j_{d}\left(\omega_{n}, \tau_{t}\right)}
$$

Thus the coupling factors $\xi_{\mathrm{DNP}}$ obtained from DNP measurements using equation (S5) and $\xi_{\text {NMRD }}$ from the results of NMRD experiments using equation (S20) are complementary parameters that allow the validation and refinement of the used models.

Employing the Rouse model, the relaxation rate of the PB block is given by:

$$
R_{1 P B}\left(\omega_{n}\right)=C_{R, P B} \tau_{S, P B} \ln \left(\frac{1}{\omega_{n} \tau_{s, P B}}\right)+C_{P B}
$$

where $C_{R, P B}$ is a prefactor which contains parameters such as the proton gyromagnetic ratio and the mean distance between protons; $C_{P B}$ is an offset constant relating to fast intrasegment motions, and $\tau_{S, P B}$ corresponds to the segmental relaxation time. In contrast, NMRD of the PS blocks in SBS solution needs to be expressed as a combination of Rouse and Lorentzian components, the latter being related to the rotation of the phenyl groups of the PS blocks:

$$
R_{1 P S}\left(\omega_{n}\right)=C_{L, P S}\left(\frac{\tau_{L, P S}}{1+\left(\omega_{n} \tau_{L, P S}\right)^{2}}+\frac{4 \tau_{L, P S}}{1+\left(\omega_{n} \tau_{L, P S}\right)^{2}}\right)+C_{R, P S} \tau_{S, P S} \ln \left(\frac{1}{\omega_{n} \tau_{S, P S}}\right)+C_{P S}
$$

where $C_{R, P S}, C_{P S}$ and $\tau_{S, P S}$ have the meaning equivalent to those in equation (S21) for the PS blocks dynamics, while $C_{L, P S}$ and $\tau_{L, P S}$ are a constant and the correlation time for the Lorentzian component of relaxation dispersion. 


\section{Experimental results}

Original CW EPR spectra are presented on Figure S1.

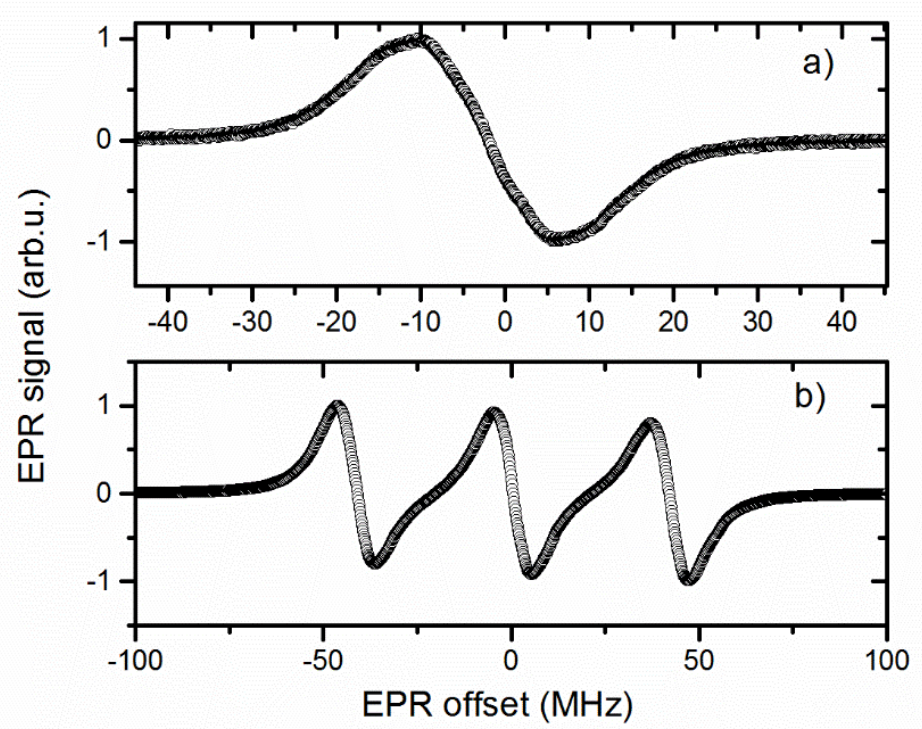

Figure S2. EPR spectra of BDPA (a) and TEMPO (b) in 10 vol.\% of SBS in deuterated cyclohexane solution at $293 \mathrm{~K}$.

Power dependencies of DNP enhancement factor of SBS solutions with TEMPO and BDPA at different concentrations ( 10 and $20 \mathrm{mM}$ of TEMPO and 2.5 and $11 \mathrm{mM}$ of BDPA) are presented on Figure S2. The results of simulation using (S4) are presented in Table S1.

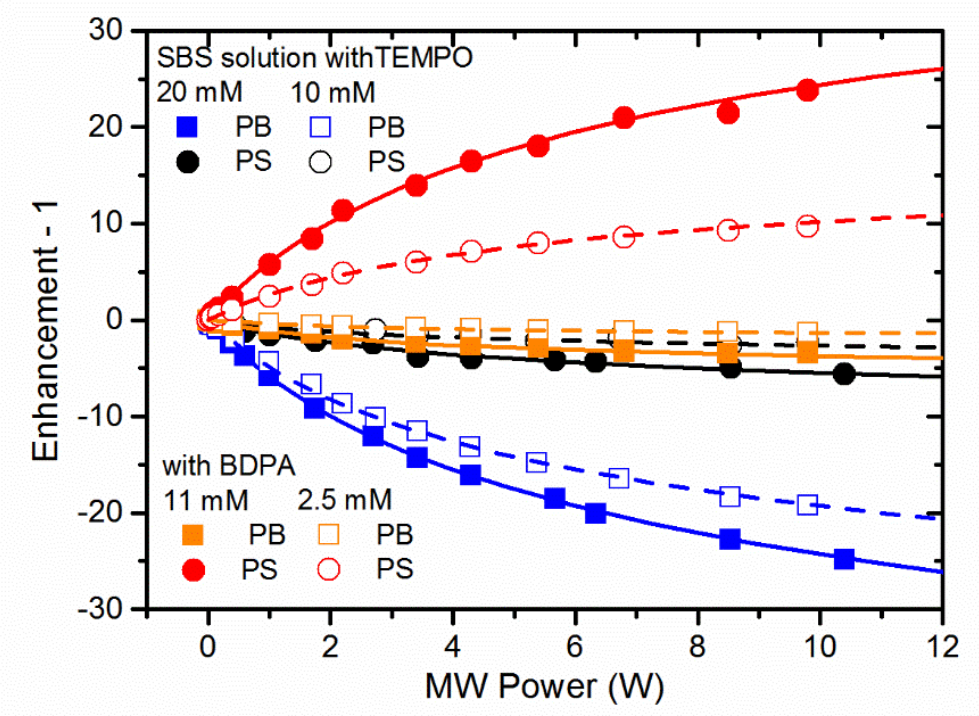

Figure S3. Power dependence of DNP enhancement of 10 vol. \% SBS in $\mathrm{C}_{6} \mathrm{D}_{12}$ with TEMPO and BDPA radicals at different concentration. Lines correspond to simulations using equation (S4).

The correlation plot of the recovered by $5 \& 10$ approach $R_{1,5 \nLeftarrow 10}$ values obtained at different magnetic fields, using a combination of NMRD of SBS with two different radical concentrations, and directly measured $R_{1, \text { exp }}$ values of pure SBS solution without radicals is presented in Figure S4. The remarkable correlation with high Pearson correlation coefficients (see Table S4) was obtained showing the high potential of using $5 \& 10$ approach. 

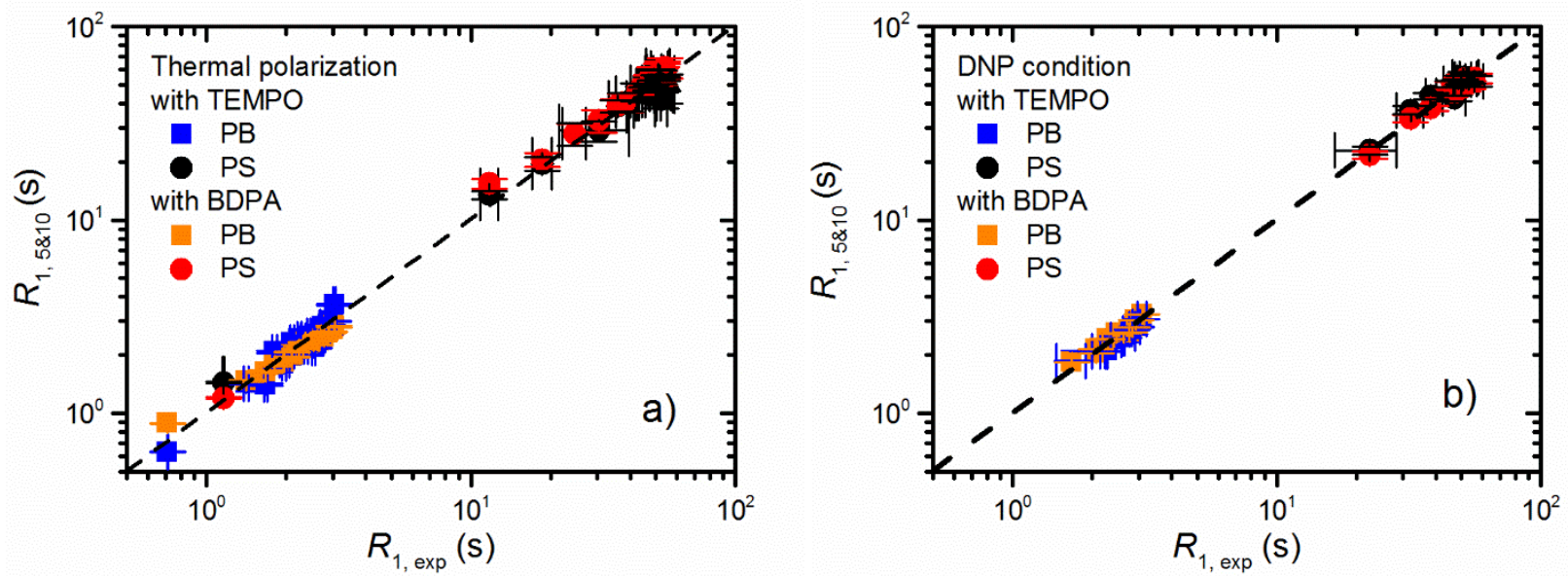

Figure S4. Correlation of the recovered by $5 \& 10$ approach $R_{1,5 \& 10}$ values on directly measured $R_{1, \exp }$ values of pure SBS solution without radicals obtained at different magnetic fields at thermal polarization (a) and DNP conditions (b). Symbols represent relaxation data where each measurement has been taken at the same magnetic field values under each condition indicated by the labels. 
Table S1. Results of simulation DNP spectra by (S4)

\begin{tabular}{|c|c|c|c|c|c|c|c|c|c|c|}
\hline \multirow{2}{*}{$\begin{array}{l}\text { Radi- } \\
\text { cal }\end{array}$} & \multirow[t]{2}{*}{ Conc.(mM) } & \multirow[t]{2}{*}{ Block } & \multicolumn{5}{|c|}{ Input parameters } & \multicolumn{3}{|c|}{ Adjusted parameters } \\
\hline & & & $\Gamma_{\mathrm{g}}, \mathrm{MHz}$ & $\Gamma_{\mathrm{l}}, \mathrm{MHz}$ & Q & $f$ & $R_{\ln , \mathrm{s}}$ & $\xi_{\mathrm{DNP}}{ }^{*}$ & $n^{-1} 4|q|^{2}, \times 10^{5}$ & $p$ \\
\hline \multirow{4}{*}{$\begin{array}{l}\mathrm{T} \\
\mathrm{E} \\
\mathrm{M} \\
\mathrm{P} \\
\mathrm{O}\end{array}$} & \multirow[t]{2}{*}{10} & $\mathrm{~PB}$ & \multirow{2}{*}{$\begin{array}{l}7.1 \\
(18.5)^{*}\end{array}$} & \multirow{2}{*}{$\begin{array}{l}11.8 \\
(22.4)^{*}\end{array}$} & \multirow[t]{2}{*}{490} & 0.56 & 0.24 & $0.12 \pm 0.02$ & - & - \\
\hline & & PS & & & & 0.29 & 0.028 & $0.02 \pm 0.01$ & - & - \\
\hline & \multirow[t]{2}{*}{20} & $\mathrm{~PB}$ & \multirow{2}{*}{$\begin{array}{l}8.7 \\
(22.4)^{*}\end{array}$} & \multirow{2}{*}{$\begin{array}{l}12.4 \\
(26.7)^{*}\end{array}$} & \multirow[t]{2}{*}{470} & 0.71 & 0.16 & $0.11 \pm 0.01$ & - & - \\
\hline & & PS & & & & 0.42 & 0.022 & $0.04 \pm 0.01$ & - & - \\
\hline \multirow{4}{*}{$\begin{array}{l}\mathrm{B} \\
\mathrm{D} \\
\mathrm{P} \\
\mathrm{A}\end{array}$} & \multirow[t]{2}{*}{2.5} & $\mathrm{~PB}$ & \multirow[t]{2}{*}{4.6} & \multirow[t]{2}{*}{3.7} & \multirow[t]{2}{*}{510} & 0.11 & 0.48 & $0.04 \pm 0.02$ & $0.4 \pm 0.2$ & $0.08 \pm 0.02$ \\
\hline & & PS & & & & 0.15 & 0.034 & - & $20 \pm 5$ & $0.10 \pm 0.02$ \\
\hline & \multirow[t]{2}{*}{11} & $\mathrm{~PB}$ & \multirow[t]{2}{*}{4.8} & \multirow[t]{2}{*}{3.9} & \multirow[t]{2}{*}{490} & 0.37 & 0.340 & $0.02 \pm 0.01$ & $0.5 \pm 0.3$ & $0.10 \pm 0.02$ \\
\hline & & PS & & & & 0.28 & 0.029 & - & $34 \pm 6$ & $0.14 \pm 0.03$ \\
\hline
\end{tabular}

${ }^{*}$ the linewidth parameters for the second component (

** the coupling factor values are presented at $293 \mathrm{~K}$ and $0.34 \mathrm{~T}$

Table S2. The fitted values of correlations times $\tau_{\mathrm{R}}, \tau_{\mathrm{L}}$ and prefactors $C_{\mathrm{R}}, C_{\mathrm{L}}$ and $C$ of models $(\mathrm{S} 20)$ and $(\mathrm{S} 21)$

\begin{tabular}{|l|l|l|l|l|l|}
\hline Block & $\tau_{s}, 10^{-9} \mathrm{~s}$ (Rouse) & $\tau_{L}, 10^{-9} \mathrm{~s}$ (Lorentzian) & $C_{\mathrm{R}}, \times 10^{-9}$ & $C_{\mathrm{L}}, \times 10^{-9}$ & $C$ \\
\hline PB & $0.25 \pm 0.02$ & - & $1.2 \pm 0.1$ & - & $0.5 \pm 0.1$ \\
\hline PS & $0.7 \pm 0.08$ & $4.9 \pm 0.4$ & $4.4 \pm 0.4$ & $1.0 \pm 0.1$ & $1.1 \pm 0.2$ \\
\hline
\end{tabular}

Table S3. The fitted values of relaxivity $r^{\text {rad }}$, correlation time $\tau_{\mathrm{t}}$ and calculated coupling factor $\xi_{\text {NMRD }}$ obtained using FFHS model (S18)

\begin{tabular}{|l|l|l|l|l|}
\hline Block & Conc., $\mathrm{mM}$ & $\hat{r}^{\mathrm{rad}}, 1 / \mathrm{s}$ & $\tau_{t}, 10^{-9} \mathrm{~s}$ & $\xi_{\mathrm{NMRD}}{ }^{*}$ \\
\hline \multicolumn{5}{|l|}{ with TEMPO radical } \\
\hline PB & $20 \& 10$ & $0.069 \pm 0.009$ & $0.11 \pm 0.01$ & $0.11 \pm 0.03$ \\
\hline PS & $20 \& 10$ & $0.31 \pm 0.02$ & $0.32 \pm 0.06$ & $0.02 \pm 0.01$ \\
\hline \multicolumn{5}{|l|}{ with BDPA radical } \\
\hline PB & $11 \& 2.5$ & $0.032 \pm 0.02$ & $0.21 \pm 0.05$ & $0.03 \pm 0.01$ \\
\hline PS & $11 \& 2.5$ & $0.41 \pm 0.04$ & $6.1 \pm 0.7$ & $(1 \pm 0.5) \times 10^{-3}$ \\
\hline
\end{tabular}

* the coupling factor values are presented at $293 \mathrm{~K}$ and $0.34 \mathrm{~T}$

Table S4. Pearson correlation coefficients obtained from correlation plots of the recovered $T_{1,5 \& 10}$ (at DNP and thermal poalrization) values and directly measured $T_{1, \text { exp }}$ values(see Figure S3)

\begin{tabular}{|l|l|l|l|l|}
\hline \multirow{2}{*}{ Polarization } & \multicolumn{2}{|l|}{ TEMPO } & \multicolumn{2}{l|}{ BDPA } \\
\cline { 2 - 5 } & PB block & PS block & PB block & PS block \\
\hline Thermal & 0.98 & 0.99 & 0.99 & 0.99 \\
\hline DNP & 0.97 & 0.94 & 0.97 & 0.98 \\
\hline
\end{tabular}




\section{REFERENCES FOR SUPPLEMENTARY INFORMATION:}

(1) Jeffries, C.D., Polarization of Nuclei by Resonance Saturation in Paramagnetic Crystals, Phys. Rev. 1957, 106, 164-165.

(2) Hausser, K. H.; Stehlik, D., Dynamics Nuclear Polarization in Liquid, Adv. Magn. Reson. 1968, 3, 79-139.

(3) Abragam, A.; Goldman, M., Principles of dynamic nuclear polarization, Rep. Prog. Phys. 1978, 41, 395-467.

(4) Freed, J.H., Dynamic effects of pair correlation functions on spin relaxation by translational diffusion in liquids. II. Finite jumps and independent T1 processes. J. Chem. Phys. 1978, 68, 4034-4037..

(5) Hwang, L.-P.; Freed, J.H., Dynamic effects of pair correlation functions on spin relaxation by tran slational diffusion in liquids. J. Chem. Phys. 1975, 63, 4017-4025.

(6) Hubbard, P. S., Theory of electron-nucleus Overhauser effects in liquids containing free radicals, Proc. R. Soc. London, Ser. A 1966, 291, $537-555$

(7) Ravera, E.; Luchinat, C.; Parigi, G. J., Basic facts and perspectives of Overhauser DNP NMR, Magn. Reson. 2016, $264,78-87$.

(8) Kruk, D.; Hoffmann, S. K.; Goslar, J.; Lijewski, S.; Kubica-Misztal, A.; Korpala, A.; Oglodek, I.; Kowalewski, J.; Rossler, E. A.; Moscicki, J., ESR lineshape and $1 \mathrm{H}$ spin-lattice relaxation dispersion in propylene glycol solutions of nitroxide radicals--joint analysis, J. Chem. Phys. 2013, 139 (24), 244502. 